\title{
Neural Network Solution of Inverse Problems of Geological Prospecting with Discrete Output
}

\author{
Igor Isaev, ${ }^{a, b, *}$ Ivan Obornev, ${ }^{a}$ Eugeny Obornev, ${ }^{c}$ Eugeny Rodionov, ${ }^{c}$ Mikhail \\ Shimelevich $^{c}$ and Sergey Dolenko ${ }^{a}$ \\ ${ }^{a}$ D.V. Skobeltsyn Institute of Nuclear Physics, M.V. Lomonosov Moscow State University, \\ 1(2) Leninskie gory, Moscow, 119991, Russian Federation \\ ${ }^{b}$ Kotelnikov Institute of Radioengineering and Electronics, Russian Academy of Sciences, \\ 11(7) Mokhovaya St., Moscow, 125009, Russian Federation \\ ${ }^{c}$ Sergo Ordjonikidze Russian State University for Geological Prospecting, \\ 23 Miklouho-Maclay St., Moscow, 117997, Russian Federation \\ E-mail: isaev_igorl@mail.ru, dolenko@srd.sinp.msu.ru
}

The inverse problems of exploration geophysics are to reconstruct the spatial distribution of the properties of the medium in the Earth's thickness from the geophysical fields measured on its surface. In particular, this paper deals with the problems of gravimetry, magnetometry, and magnetotelluric sounding, as well as their integration, i.e., the simultaneous use of several geophysical fields to restore the desired distribution. To implement the integration, a 4-layer 2D model was used, where the inverse problem was to determine the lower boundary of the layers, and each layer was characterized by variable values of the depth of the lower boundary along the section and fixed values of density, magnetization, and resistivity, both for the layer and for the entire data set. To implement the neural network solution of the inverse problem, a data set was generated by solving the direct problem, where for each pattern, the distribution of layer depth values was set randomly in a given range and with a given step, i.e. it took discrete values from a certain set. In this paper, we consider an approach involving the use of neural networks to solve the problem of multiclass classification, where class labels correspond to discrete values of the determined layer depths. The results of the solution are compared with the results of the solution of the same inverse problem in the formulation of the regression problem, in terms of the error in determining the depth of the layers.

The 5th International Workshop on Deep Learning in Computational Physics 28-29 June, 2021

Moscow, Russia

\footnotetext{
*Speaker
} 


\section{Introduction}

The solution of the inverse problems (IP) of exploration geophysics (EG) is aimed at searching for useful fossils by studying the structure of the near-surface layer of the earth. The general statement of the EG IP consists in reconstructing the distribution of the physical parameters of the medium in the thickness of the earth's crust from the physical fields measured on the earth's surface. In particular, in this study we consider the IP of gravimetry $(\mathrm{G})$, magnetometry $(\mathrm{M})$ and magnetotelluric sounding (MT), which consist in restoring the spatial distribution of density, magnetization, and electrical resistance of the medium in the Earth's crust by the values of gravitational, magnetic, and magnetotelluric fields, respectively. In the two-dimensional and three-dimensional cases, these IPs have no analytical solution, so they are solved numerically. These IPs are nonlinear, ill-posed or ill-conditioned problems with high dimension both at the input and at the output, which generally leads to a low quality of the solution. As an additional method for improving the quality of the solution, the present study considers the integration of geophysical methods [1-3], which consists in the simultaneous use of data of several geophysical methods.

The traditional methods for solving the EG IP include optimization methods[4] based on the multiple solution of the direct problem with the minimization of residuals in the space of the observed fields, and approximation methods, which also include matrix methods using Tikhonov regularization [5]. Optimization methods have a number of disadvantages: they are characterized by high computational costs and by the need for a good first approximation, which may be obtained using alternative measurement methods. At the same time, the main drawback of optimization methods is the need to have a correct model for solving the direct problem, in the absence of which this method is not applicable. In addition, due to the inherent incorrectness of many IP, a small residual in the space of the observed quantities does not guarantee a small residual in the space of the determined parameters (see e.g. [6]). For matrix methods based on regularization, the main difficulty is the need to choose the regularization parameter. In addition, matrix methods are linear methods, so when using them to solve nonlinear problems, it is necessary to perform nonlinear data preprocessing.

Therefore, to solve such problems, machine learning (ML) methods that are free from the above disadvantages are considered as an alternative. High computational costs when using ML methods are shifted from the stage of application of the computing system to the stage of its development, making practical use of such a system more convenient.

At the same time, when solving the RG problem, the ML methods can be used at various stages of its solution: in data preprocessing, e.g., for noise removal [7, 8]; as an independent optimization method [2, 3, 9] or as a component of optimization methods used to solve the EG problem [10]; as an independent inversion method [1,11-19]; in solving the classification problem to select a class of geological media [20]. In this study, we considered neural networks as an independent inversion method. 
The general scheme for applying the machine learning methods to solving IP of EG includes the following steps:

- Definition of a parameterization scheme with a finite number of parameters describing the sought-for distribution

- Generation of a training dataset

- Training a ML model on the training dataset

- Applying the obtained ML model to the studied data.

Generation of a training dataset involves performing the following steps for each pattern:

- Setting a random distribution of the parameters over the macro-grid determined by the parameterization scheme

- Calculation of the distribution of the parameters over the micro-grid

- Calculation of the field values by solving the direct problem using the finite difference method on the micro-grid.

In practice, in the case of high dimension of the problem being solved, it is often not a random setting of parameters that is used, but a random selection from a certain finite set of discrete values. This is also the case for the data used in this study. However, in this case, the direct application of $\mathrm{NN}$ to determine these discrete values inevitably has errors, since NN by their nature have continuous output. Therefore, in this paper we propose an approach based on the change in the initial problem statement. This approach consists in using $\mathrm{NN}$ to determine discrete values by solving a classification problem rather than a regression problem. To implement this approach, the use of "one-hot-encoding" (or 1-of-N) is considered, which is generally used in machine learning to solve problems from such areas as natural language processing [21-24], video [25] and image processing [26, 27] and generation [28], genomic data processing [29-33], and others [34, 35].

The purpose of this study is the comparison of the approach based on the direct neural network solution of the regression problem to the approach based on consideration of the discretevalued outputs, the "one-hot encoding" of these discrete outputs, and the subsequent solution of the classification problem. We also test the applicability of the integration of geophysical methods when using the classification approach in the described statement.

\section{Physical statement of the problem}

\subsection{Parameterization scheme}

In order to implement the integration of various geophysical methods, it is necessary that the determined parameters of each of the methods are the same. This approach corresponds to the geometric formulation of the problem, which consists in determination of the boundaries of geophysical objects. In particular, in this study we considered the parameterization scheme, which consists in determining the boundaries of geological layers of a layered medium. The parameterization scheme was a four-layer two-dimensional model (Fig. 1) - corresponding to a section of the Norilsk region. The first layer modeled the basalt layer, the second and fourth ones terrigenous carbonate deposits of the Tunguska series, the third one - the gabbro-dolerites massive copper-nickel-platinum ores. 


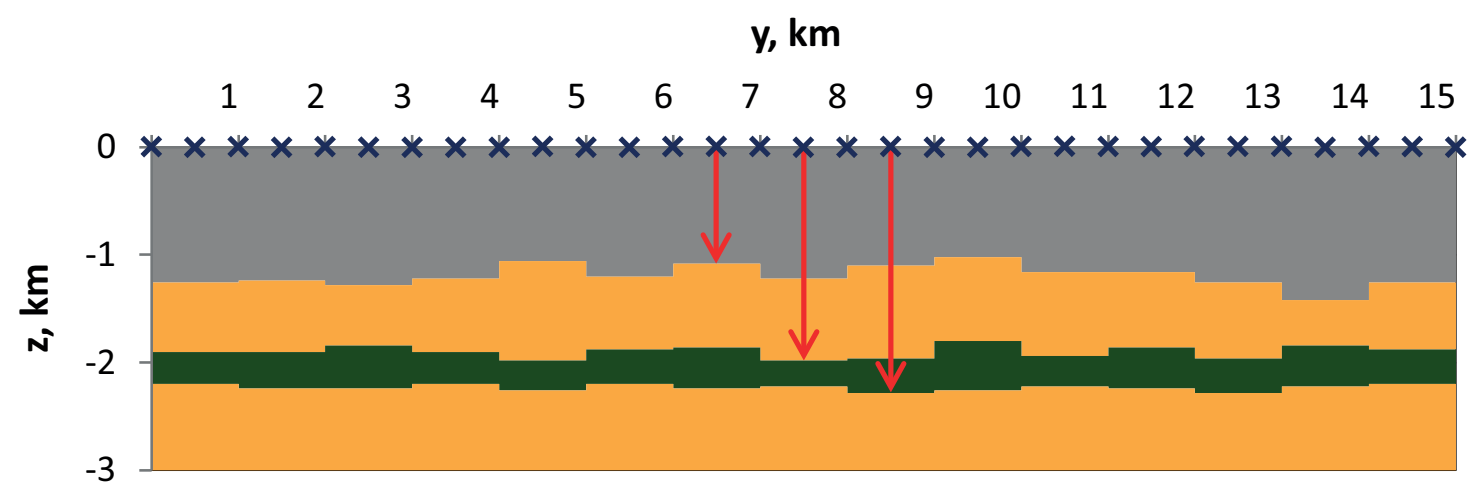

Figure 1: Parameterization scheme. Markers (x) indicate the locations of measurements of geophysical fields, arrows indicate the locations of determining the depths of the layers.

Table 1: Physical and spatial properties of the layers

\begin{tabular}{|c|c|c|c|c|c|c|}
\hline \multirow[b]{2}{*}{ Layer } & \multirow[b]{2}{*}{ Description } & \multicolumn{3}{|c|}{ Physical properties } & \multicolumn{2}{|c|}{ Spatial properties } \\
\hline & & $\begin{array}{l}\text { Density } \\
\sigma \\
\mathrm{kg} / \mathrm{m}^{3}\end{array}$ & $\begin{array}{c}\text { Magnetization } \\
\mu, \\
\mathrm{A} / \mathrm{m}\end{array}$ & $\begin{array}{c}\text { Resistivity } \\
\rho, \\
\Omega \cdot \mathrm{m}\end{array}$ & $\begin{array}{c}\text { Lower } \\
\text { bound, } \\
\min -\max , \\
\mathrm{km}\end{array}$ & $\begin{array}{c}\text { Thickness, } \\
\text { min - max, } \\
\text { km }\end{array}$ \\
\hline 1 & Basalt & 2800 & 3.0 & 2000 & $1.00-1.48$ & $1.00-1.48$ \\
\hline 2 & $\begin{array}{l}\text { Terrigenous } \\
\text { carbonate deposits of } \\
\text { the Tunguska series }\end{array}$ & 2550 & 0.5 & 100 & $1.80-1.98$ & $0.32-0.98$ \\
\hline 3 & $\begin{array}{l}\text { Gabbro-dolerites } \\
\text { massive copper- } \\
\text { nickel-platinum ores }\end{array}$ & 3000 & 0.9 & 1000 & $2.20-2.28$ & $0.22-0.48$ \\
\hline 4 & $\begin{array}{l}\text { Terrigenous } \\
\text { carbonate deposits of } \\
\text { the Tunguska series }\end{array}$ & 2550 & 0.5 & 100 & - & - \\
\hline
\end{tabular}

The dimension of the section was $15 \mathrm{~km}$ wide and $3 \mathrm{~km}$ deep. The physical field measurement step is $0.5 \mathrm{~km}-\mathrm{a}$ total of 31 measurement points along the profile. The discreteness of changing the boundaries of geological layers is $1 \mathrm{~km}-$ a total of 15 depth values for each layer. In this problem, the values of the depths of the lower boundaries of the three upper layers were determined. Each layer was characterized by fixed values of density, magnetization, and resistivity, which did not change within the layer, and which were the same across the entire data set. The physical characteristics of the second and the fourth layers were the same. The values of the physical and spatial characteristics of the layers are shown in Table 1. The discreteness of changing the values of depth was $0.02 \mathrm{~km}$. 


\subsection{Data}

For each pattern of the original data set, the layer depth values were set randomly in the ranges shown in Table 1. Further, the direct problem was solved by finite-difference methods for each of the selected geophysical methods.

The input dimension of the problem was:

- Gravimetry: 1 field component $* 31$ measurement point (picket) $=31$ feature

- Magnetometry: 1 field component $* 31$ picket $=31$ feature

- MTS: 2 field components $* 1$ frequency $* 31$ picket $=62$ features

The output dimension of the problem was:

- 3 layers $* 15$ values of layer boundary depth $=45$ parameters.

The number of possible discrete values of the output parameters was $25,10,5$ for the 1 st, 2 nd and 3rd layers, respectively.

A total of 30000 patterns were calculated.

\section{Methodical statement of the problem}

\subsection{Regression approach}

Regression approach consists in training the neural networks for direct determination of the parameter values. However, neural networks by their nature have continuous output, therefore, when they are used to determine discrete values, the solution inevitably has errors, which may be eliminated by rounding of the solution, or by transfer to the classification approach.

It should also be noted that one of the methods that may reduce the error for the really continuously valued output is preprocessing of the initial data by discretization of the outputs. If the discretization step is less or equal to the characteristic error of the output values, the changes introduced by discretization of the output values in the training set, and by rounding of the output values of the NN to these discrete values, may result in lower total output error. Within this approach, transfer from the solution of the regression problem to the solution of classification problem may additionally improve the results. This general approach will not be tested in this study, but we consider it as the next step in the development and testing of the discretization/classification approach.

Thus, the reference regression approach used in this study consists in direct consideration of the NN output values as the solution of the problem, and in their comparison to the desired output values without taking into account that these desired values in fact belong to a discrete set.

\subsection{Classification approach with one-hot-encoding}

Classification approach consists in discretization of the determined parameter, its "one-hot encoding", and subsequent solution of the classification problem. The size of the vector encoding the determined parameter is equal to the number of discrete values that the determined parameter can take. In the case considered in this study, this number of discrete values is equal to the number of different values of the layer boundary depth used in the calculation of the training set patterns by solving the direct problem. 
All the positions in this encoding vector are set to "0" except for one position corresponding to the specific desired discrete parameter value, which is set to "1". To solve the multiclass classification problem, we also use neural networks, where each position in the encoding vector corresponds to a separate output of the neural network. The solution (the value selected by the classification procedure) corresponds to the output of neural network with maximum amplitude among all its outputs.

\subsection{Reducing the output dimension}

To reduce the output dimension of the problem, autonomous determination $[13,14]$ of parameters was used, which consists in individual determination of each parameter by training a separate NN. When using the regression approach, each such $\mathrm{NN}$ has a single output. When using the classification approach, the number of the $\mathrm{NN}$ outputs is equal to the number of discrete values of the determined parameter. In this case, the $\mathrm{NN}$ had 25,10 or 5 outputs for the parameters of the 1st, 2nd, 3rd layers, respectively.

\subsection{Integrating geophysical methods and input dimension}

When integrating geophysical methods, the data of two or three geophysical methods were simultaneously fed to the input of the NN. For individual use of the data of the gravimetry and magnetometry methods, the NN input was fed with 31 features, for individual use of MTS data with 62 features, for simultaneous use of data of two geophysical methods - with 62 or 93 features, for simultaneous use of data from all the three methods - 124 features.

\subsection{Datasets}

The original data set was divided into training, validation, and test sets in the ratio of 70:20:10. The dimension of the sets was 21000,6000 , and 3000 patterns, respectively.

\subsection{Neural networks}

The type of NN used was the multi-layer perceptron. The architecture used had a single hidden layer with 32 neurons in it. Activation function of the output layer was linear for the regression approach and logistic for the classification approach. To prevent overtraining, early stopping by the validation dataset was used. Training stops after 500 epochs with no improvement on the validation set.

To reduce the factor imposed by the influence of the initialization of weights on the training of NN, 5 networks were trained for each case under consideration, and the statistical indicators of their application were averaged. 


\section{Results}

Fig. 2 shows an example of the amplitudes of the neural network outputs when using the classification approach to determine the boundary depths of different layers using different combinations of the integrated input data for a separate sample. One can see that the use of the integration of geophysical methods increases the stability of the neural network classification when using the classification approach.

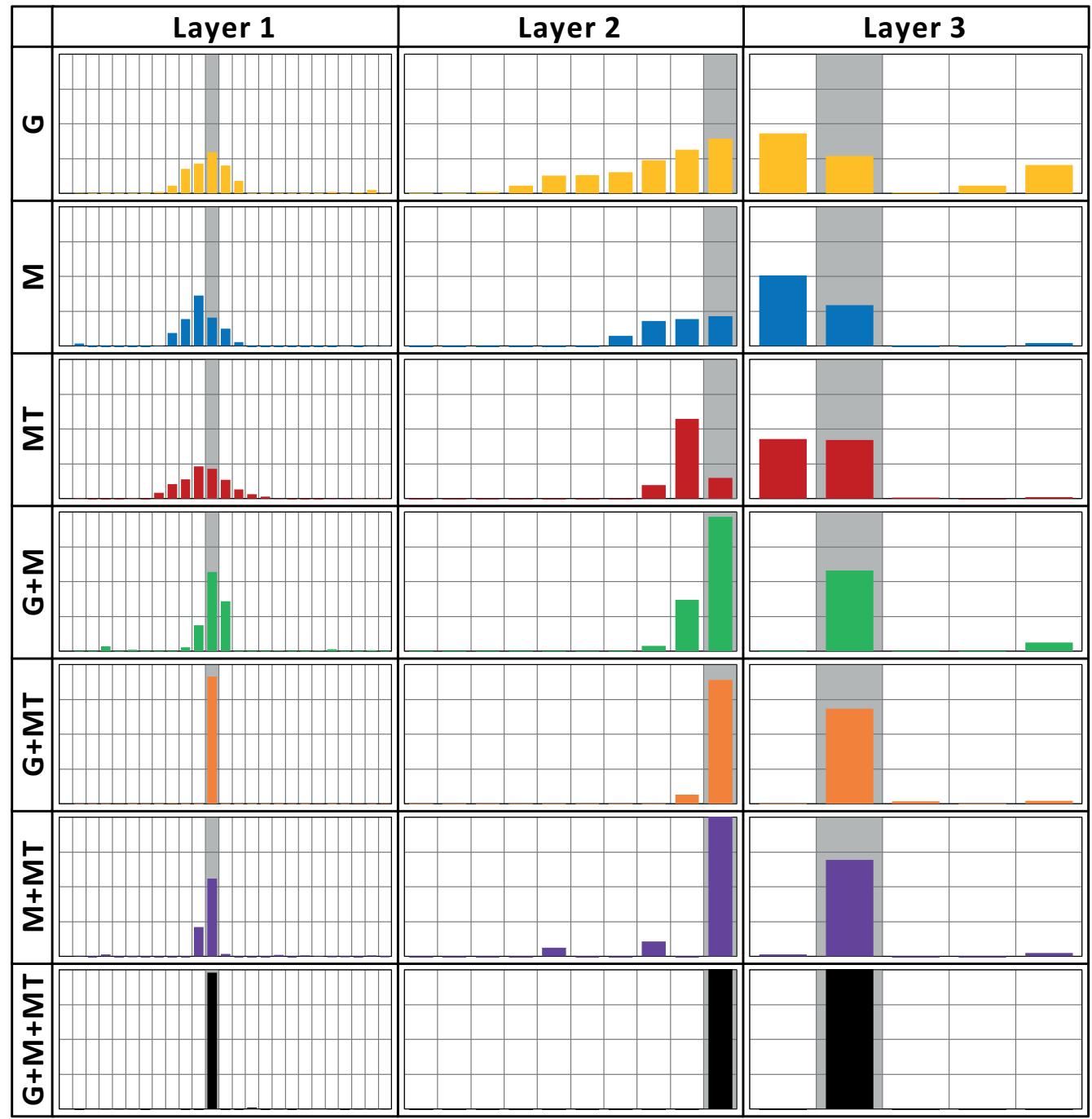

Figure 2: An example of the amplitudes of the neural network outputs when using the classification approach to determine the boundary depths of different layers using different input data for a separate sample. Each column denotes a separate output of the neural network. The gray color indicates the true answer. Vertical axis scale in each diagram is from 0 to 1 . G, M, MT - individual use of gravimetry, magnetometry and MTS data; G+M, G+MT, M+MT, G+M+MT - simultaneous use of data from several geophysical methods (integration of methods). 
Fig. 3 shows the histogram of errors when using the classification and regression approaches to determine the boundary depths of different layers using different input data. One can see that the use of the integration of geophysical methods reduces the number of errors with large values for all layers and for both approaches. In some cases, the classification approach gives a better result than the regression approach. Also one can see that it may be possible to further reduce the errors of the regression approach by rounding the answer to the nearest discrete value.

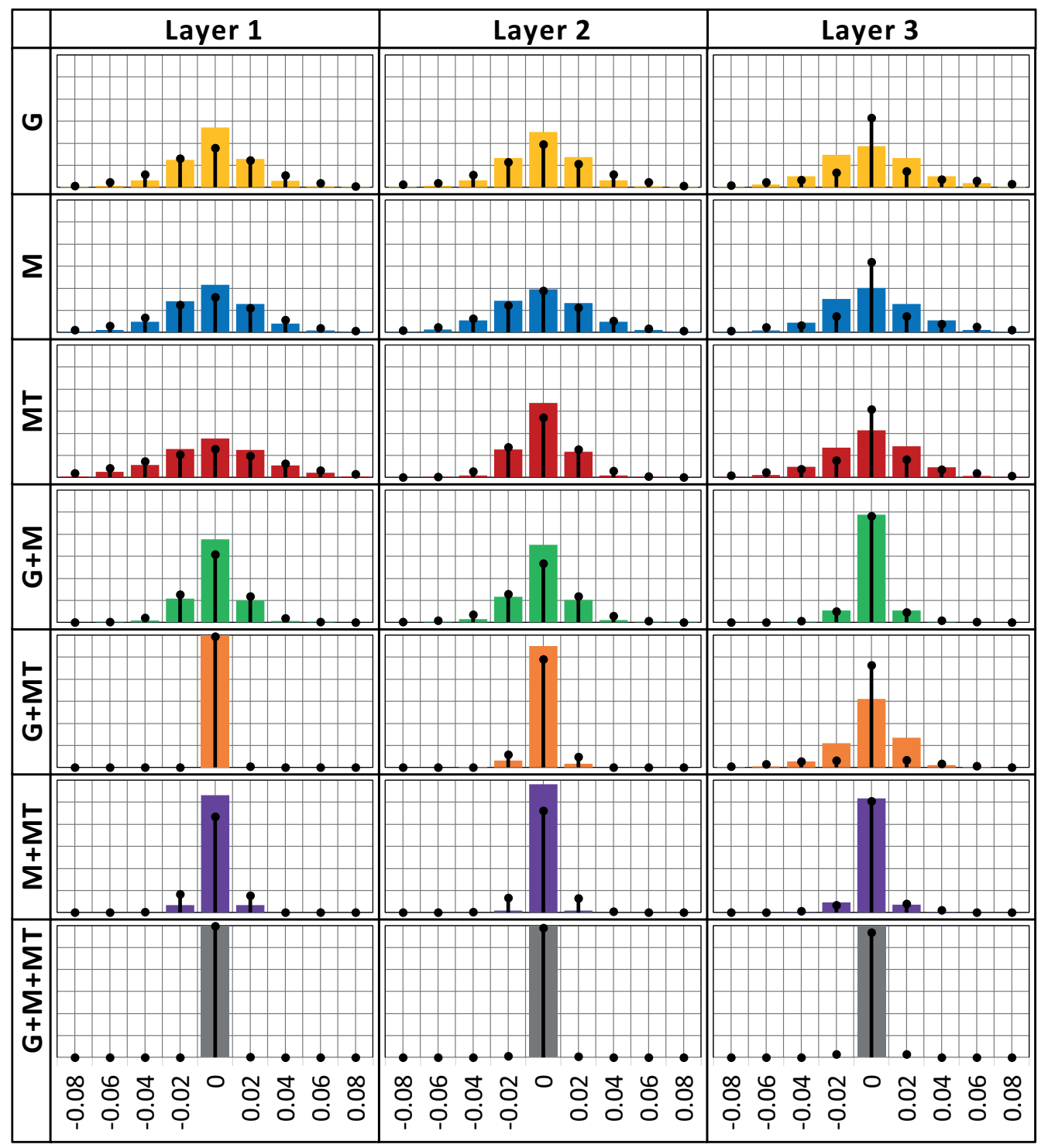

Figure 3: Histogram of errors when using the classification (markers) and regression (bars) approaches to determine the boundary depths of different layers using different input data averaged over 5 neural networks. Vertical axis scale in each diagram is from 0 to 3000 (the size of the test dataset). The position of the markers corresponds to the discretization step of the output values, since the errors take discrete values. The bin width also corresponds to discretization step of the output values. The position of the bin centers coincides with the position of the markers. G, M, MT - individual use of gravimetry, magnetometry and MTS data; $\mathrm{G}+\mathrm{M}, \mathrm{G}+\mathrm{MT}, \mathrm{M}+\mathrm{MT}, \mathrm{G}+\mathrm{M}+\mathrm{MT}-$ simultaneous use of data of several geophysical methods (integration of methods). 
The overall results for regression and classification approaches and for various sets of input data are presented in Fig. 4. As an indicator of the quality of the solution, we used the relative error calculated as the mean absolute error (MAE) normalized by the range of change of the determined parameter. For all the three layers and for both approaches, simultaneous use of data of any two geophysical methods reduces the error compared to the individual use of data of any of them. The best result is shown by simultaneous use of data of all the three geophysical methods.

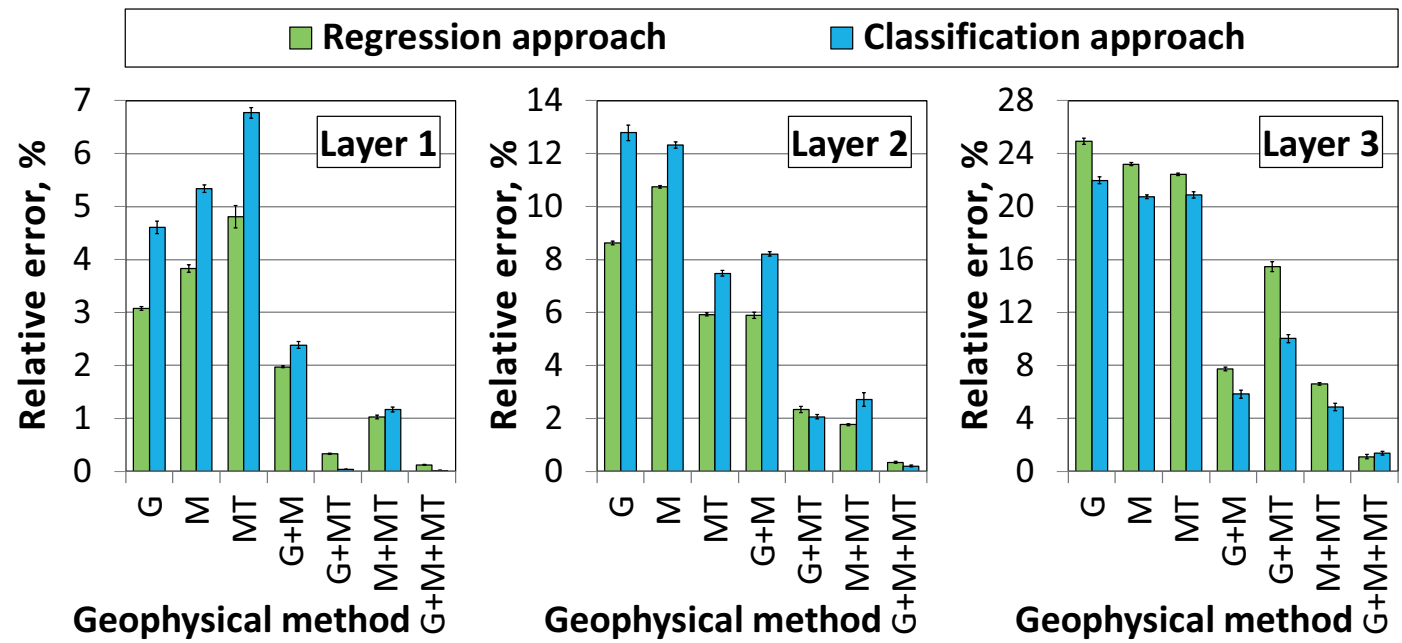

Figure 4: Dependence of the quality of the solution on the integration of the input data for regression and classification approaches.

In some cases, the classification approach gives a better result than the regression approach. For the 1st and the 2nd layers, this result is observed in the case of integration of gravimetry and magnetotellurics, and with integration of all the three geophysical methods. For the 3rd layer, this result is observed in most cases.

\section{Conclusion}

Based on the results of this study, the following conclusions can be drawn:

- The classification approach was capable to give better results than the regression approach in the following cases:

- For the 1st and the 2nd layer with integration of gravimetry and magnetotellurics, and with integration of all the three geophysical methods

- For the 3rd layer in most cases.

- Integration of geophysical methods improves the results for all layers, both for the regression approach and for the classification approach:

$\circ$ The use of the integration of geophysical methods increases the stability of the neural network classification when using the classification approach

- Simultaneous use of data of any two geophysical methods reduces the error compared to the individual use of data of any one of them.

- The best result is shown by the simultaneous use of data of all the three geophysical methods. 
Thus, in the present study, the applicability of the classification approach based on "one-hotencoding" to improve the quality of the solution to the inverse problem of exploration geophysics has been demonstrated for the dataset in which the desired output accepts values from a discrete set. Also, the efficiency of the integration of geophysical methods to improve the quality of the solution has been confirmed also for the case of using the classification approach.

Future studies should examine the efficiency of the classification approach for the case when the outputs accept continuous values from a defined range, within the sequence "discretization" "one-hot encoding" - "classification".

\section{Acknowledgments}

This study has been performed at the expense of the grant of the Russian Science Foundation (project no. 19-11-00333).

\section{References}

[1] E. Obornev, I. Obornev, E. Rodionov, and M. Shimelevich, Application of Neural Networks in Nonlinear Inverse Problems of Geophysics, Computational Mathematics and Mathematical Physics 60.6 (2020): 1025-1036. doi: 10.1134/S096554252006007X.

[2] İ. Akca, T. Günther, M. Müller-Petke, A. T. Başokur, and U. Yaramanci, Joint parameter estimation from magnetic resonance and vertical electric soundings using a multi-objective genetic algorithm, Geophysical Prospecting 62.2 (2014): 364-376. doi:10.1111/1365-2478.12082.

[3] E. Roux, M. Moorkamp, A. G. Jones, M. Bischoff, B. Endrun, S. Lebedev, and T. Meier, Joint inversion of long-period magnetotelluric data and surface-wave dispersion curves for anisotropic structure: Application to data from Central Germany, Geophysical Research Letters 38.5 (2011): L05304. doi:10.1029/2010GL046358.

[4] M. S. Zhdanov, Inverse Theory and Applications in Geophysics, 2nd ed., Elsevier, Amsterdam, 2015.

[5] M. S. Zhdanov, Geophysical electromagnetic theory and methods, volume 43 of Methods in Geochemistry and Geophysics, Elsevier, Amsterdam, 2009.

[6] I. Isaev, and S. Dolenko, Comparative Analysis of Residual Minimization and Artificial Neural Networks as Methods of Solving Inverse Problems: Test on Model Data, in: A. Samsonovich, V. Klimov, G. Rybina (eds) Biologically Inspired Cognitive Architectures (BICA) for Young Scientists, volume 449 of Advances in Intelligent Systems and Computing, Springer, Cham, 2016, pp. 289-295. doi:10.1007/978-3-319-32554-5_37.

[7] C. Manoj, and N. Nagarajan, The application of artificial neural networks to magnetotelluric time-series analysis, Geophysical Journal International 153.2 (2003): 409-423. doi:10.1046/j.1365-246X.2003.01902.x. 
[8] X. Wu, G. Xue, P. Xiao, J. Li, L. Liu, and G. Fang, The removal of the high-frequency motioninduced noise in helicopter-borne transient electromagnetic data based on wavelet neural network, Geophysics 84.1 (2019): K1-K9. doi:10.1190/geo2018-0120.1.

[9] S. Yuan, S. Wang, and N. Tian, Swarm intelligence optimization and its application in geophysical data inversion, Applied Geophysics 6.2 (2009): 166-174. doi:10.1007/s11770-0090018-x.

[10] D. Conway, B. Alexander, M. King, G. Heinson, and Y. Kee, Inverting magnetotelluric responses in a three-dimensional earth using fast forward approximations based on artificial neural networks, Computers \& Geosciences 127 (2019): 44-52. doi:10.1016/j.cageo.2019.03.002.

[11] M. A. Al-Garni, Inversion of residual gravity anomalies using neural network, Arabian Journal of Geosciences 6 (2013): 1509-1516. doi:10.1007/s12517-011-0452-y.

[12] M. A. Al-Garni, Interpretation of some magnetic bodies using neural networks inversion, Arabian Journal of Geosciences 2 (2009): 175-184. doi:10.1007/s12517-008-0026-9.

[13] S. Dolenko, I. Isaev, E. Obornev, I. Persiantsev, and M. Shimelevich, Study of Influence of Parameter Grouping on the Error of Neural Network Solution of the Inverse Problem of Electrical Prospecting, in: L. Iliadis, H. Papadopoulos, C. Jayne (eds) Engineering Applications of Neural Networks, EANN 2013, volume 383 of Communications in Computer and Information Science, Springer, Berlin, Heidelberg, 2013, pp. 81-90. doi:10.1007/978-3-642-41013-0_9.

[14] I. Isaev, E. Obornev, I. Obornev, M. Shimelevich, and S. Dolenko, Increase of the Resistance to Noise in Data for Neural Network Solution of the Inverse Problem of Magnetotellurics with Group Determination of Parameters, In: A. Villa, P. Masulli, A. Pons Rivero (eds) Artificial Neural Networks and Machine Learning - ICANN 2016, ICANN 2016, volume 9886 of Lecture Notes in Computer Science, Springer, Cham, 2016, pp. 502-509. doi:10.1007/978-3319-44778-0_59.

[15] I. Isaev, and S. Dolenko, Adding Noise During Training as a Method to Increase Resilience of Neural Network Solution of Inverse Problems: Test on the Data of Magnetotelluric Sounding Problem, In: B. Kryzhanovsky, W. Dunin-Barkowski, V. Redko (eds) Advances in Neural Computation, Machine Learning, and Cognitive Research, NEUROINFORMATICS 2017, volume 736 of Studies in Computational Intelligence, Springer, Cham, 2018, pp. 9-16. doi:10.1007/978-3-319-66604-4_2.

[16] V. Spichak, and I. Popova, Artificial neural network inversion of magnetotelluric data in terms of three-dimensional earth macroparameters, Geophysical Journal International 142.1 (2000): 15-26. doi:10.1046/j.1365-246x.2000.00065.x.

[17] V. Spichak, K. Fukuoka, T. Kobayashi, T. Mogi, I. Popova, and H. Shima, ANN reconstruction of geoelectrical parameters of the Minou fault zone by scalar CSAMT data, Journal of Applied Geophysics 49.1-2 (2002): 75-90. doi:10.1016/S0926-9851(01)00100-8. 
[18] M. Montahaei, and B. Oskooi, Magnetotelluric inversion for azimuthally anisotropic resistivities employing artificial neural networks, Acta Geophysica 62.1 (2014): 12-43. doi:10.2478/s11600-013-0164-7.

[19] I. Isaev, E. Obornev, I. Obornev, E. Rodionov, M. Shimelevich, V. Shirokiy, and S. Dolenko, Using Domain Knowledge for Feature Selection in Neural Network Solution of the Inverse Problem of Magnetotelluric Sounding, in: A. V. Samsonovich, R. R. Gudwin, A. S. Simões (eds) Brain-Inspired Cognitive Architectures for Artificial Intelligence: BICA*AI 2020, BICA 2020, volume 1310 of Advances in Intelligent Systems and Computing, Springer, Cham, 2021, pp. 115-126. doi:10.1007/978-3-030-65596-9_15.

[20] I. Isaev, E. Obornev, I. Obornev, M. Shimelevich, and S. Dolenko, Neural Network Recognition of the Type of Parameterization Scheme for Magnetotelluric Data, In: B. Kryzhanovsky, W. Dunin-Barkowski, V. Redko, Y. Tiumentsev (eds) Advances in Neural Computation, Machine Learning, and Cognitive Research II, NEUROINFORMATICS 2018, volume 799 of Studies in Computational Intelligence, Springer, Cham, 2019, pp. 176-183. doi:10.1007/978-3-03001328-8_19.

[21] X. Zhang, J. Zhao, and Y. LeCun, Character-level convolutional networks for text classification, Advances in neural information processing systems 28 (2015): 649-657.

[22] C. Braud, and P. Denis, Comparing word representations for implicit discourse relation classification, in: Empirical Methods in Natural Language Processing (EMNLP 2015), 2015.

[23] Y. Zhang, and B. Wallace, A sensitivity analysis of (and practitioners' guide to) convolutional neural networks for sentence classification, arXiv preprint arXiv: 1510.03820 (2015).

[24] X. Zhang, and Y. LeCun, Which encoding is the best for text classification in chinese, english, japanese and korean?, arXiv preprint arXiv:1708.02657 (2017).

[25] X. Wang, and Q. Ji, Video event recognition with deep hierarchical context model, in: Proceedings of the IEEE Conference on Computer Vision and Pattern Recognition (CVPR), 2015, pp. 4418-4427.

[26] M. D. Hasan, S. Ahmed, Z. M. Abdullah, M. Monirujjaman Khan, D. Anand, A. Singh, M. AlZain, and M. Masud, Deep Learning Approaches for Detecting Pneumonia in COVID19 Patients by Analyzing Chest X-Ray Images, Mathematical Problems in Engineering 2021 (2021). doi:10.1155/2021/9929274.

[27] C. Yu, J. Wang, C. Gao, G. Yu, C. Shen, and N. Sang, Context prior for scene segmentation, in: Proceedings of the IEEE/CVF Conference on Computer Vision and Pattern Recognition (CVPR), 2020, pp. 12416-12425.

[28] A. V. D. Oord, N. Kalchbrenner, O. Vinyals, L. Espeholt, A. Graves, and K. Kavukcuoglu, Conditional image generation with pixelCNN decoders, arXiv preprint arXiv: 1606.05328 (2016). 
[29] G. L. Bosco, and M. A. Di Gangi, Deep learning architectures for DNA sequence classification, in: A. Petrosino, V. Loia, W. Pedrycz (eds) Fuzzy Logic and Soft Computing Applications, volume 10147 of Lecture Notes in Computer Science, Springer, Cham, 2020, pp. 162-171. doi:10.1007/978-3-319-52962-2_14.

[30] A. C. H. Choong, and N. K. Lee, Evaluation of convolutionary neural networks modeling of DNA sequences using ordinal versus one-hot encoding method, in: 2017 International Conference on Computer and Drone Applications (IConDA), 2017, pp. 60-65. doi:10.1109/ICONDA.2017.8270400.

[31] M. Tahir, H. Tayara, and K. T. Chong, iDNA6mA (5-step rule): Identification of DNA N6-methyladenine sites in the rice genome by intelligent computational model via Chou's 5-step rule, Chemometrics and Intelligent Laboratory Systems 189 (2019): 96-101. doi:10.1016/j.chemolab.2019.04.007.

[32] Z. Lv, H. Ding, L. Wang, and Q. Zou, A convolutional neural network using dinucleotide onehot encoder for identifying DNA N6-methyladenine sites in the rice genome, Neurocomputing 422 (2021): 214-221. doi:10.1016/j.neucom.2020.09.056.

[33] Q. Zhang, Z. Shen, and D. S. Huang, Modeling in-vivo protein-DNA binding by combining multiple-instance learning with a hybrid deep neural network, Scientific reports 9.8484 (2019). doi:10.1038/s41598-019-44966-x.

[34] Y. Qu, H. Cai, K. Ren, W. Zhang, Y. Yu, Y. Wen, and J. Wang, Product-based neural networks for user response prediction, in: 2016 IEEE 16th International Conference on Data Mining (ICDM), 2016, pp. 1149-1154. doi:10.1109/ICDM.2016.0151.

[35] J. Li, Y. Si, T. Xu, and S. Jiang, Deep convolutional neural network based ECG classification system using information fusion and one-hot encoding techniques, Mathematical Problems in Engineering 2018.7354081 (2018). doi:10.1155/2018/7354081. 